\title{
Effect of non-isothermal flow on chloride ion transport in saturated concrete
}

\author{
Chen Peng ${ }^{1, *}$, Qing $\mathrm{Wu}^{1}$, and Jun $\mathrm{Xu}^{1,2}$ \\ ${ }^{1}$ College of Civil Engineering and Architecture, Jiangsu University of Science and Technology, Zhenjiang, China \\ ${ }^{2}$ School of Materials Science and Engineering, Southeast University, Nanjing, China
}

\begin{abstract}
The presence of a temperature difference changes the mode of motion of the fluid. The macroscopic motion of the fluid causes the relative displacement between the various parts of the fluid, which in turn affects the transfer of temperature in the medium. To investigate the effect of temperature transfer coupled fluid motion on chloride ion transport in concrete, a mesoscopic model of chloride ion transport in non-isothermal flow of concrete was established and compared with experimental data. Based on the finite element software, the influence of temperature transfer on the internal fluid motion and chloride ion transport of concrete was analyzed. And the effect of convective motion of fluid on the temperature transfer in concrete was studied. It is found that when the heating time is the same, the dynamic viscosity of the fluid in the concrete decreases with the increase of temperature; the chloride ion concentration increases with the increase of temperature at the same depth of concrete; when considering the influence of laminar flow, the temperature is transmitted faster in the concrete and exhibits uneven diffusion from high temperature to low temperature; non-isothermal flow promotes the diffusion of chloride ions in concrete, and the higher the temperature.
\end{abstract}

\section{Introduction}

The transmission of chloride ions in concrete is the first stage of the service life of reinforced concrete in marine engineering, and it is also a key factor affecting the total service life of the concrete. Chloride ion diffusion erosion will cause corrosion of the steel bar inside the concrete, and the corrosion of the steel bar will reduce the crosssectional area of the steel bar and reduce its ability to bond with the concrete, thereby further reducing the sustainability of the structure [1-3].

For the transport of chloride ions in concrete, researchers at home and abroad are changing with each passing day. Fan et al. [4] analyzed the raw materials and environmental conditions of exposed concrete, measured the chloride ion distribution in the core sample and the test specimen in the laboratory, and evaluated the chloride ion diffusion coefficient based on the chloride ion distribution curve. The influence of material and environmental factors on the distribution of chloride ions was analyzed. Hou et al. [5] carried out an experimental study on the effect of chloride ion corrosion on the bonding performance between steel bars and steel fiber reinforced concrete. The test parameters included the corrosion ratio, fiber fraction, and cover thick-ness-rebar diameter ratio (c / d). And they com-pared the failure mode, bond-slip curve, and bond strength, a combined strength model that considers the combined effect of corrosion ratio, fiber fraction, and c/d ratio was proposed. Abbas et al. [6] have utilized ultrasonic waves to detect the concentration of chlorideions in cement slurries with supplementary cementitious materials. One should note that an exponential relationship exists between the energy/amplitude weighted average frequency and chemically combined chloride content in the cement slurry.

Different external environments have different effects on the durability degradation of concrete. The temperature of the service environment has time-varying properties, and there is a temperature difference between inside and outside, which will affect the chloride ion transmission in concrete. Quan \& Soive [7] proposed a new physical and chemical-based model that considers thermodynamic equilibrium, kinetics, and surface complexation to predict the process of chloride ions entering saturated concrete. Wang \& Ueda [8] established a meso-scale truss network model to predict the chloride diffusion in concrete. This model takes into account the dependence of chloride diffusion coefficient on the retention temperature in the mor-tar and interface transition zone. Zhao et al. [9] constructed a concrete model considering the inter-face area of aggregate and slurry aggregates, established a chloride ion transmission model affected by environmental temperature and humidity, and analyzed the chloride ion of concrete and materials based on COMSOL numerical simulation software. The influence of transmission is verified by experiments. Khatib \& Mangat [10] researched the in-fluence of hightemperature and low-humidity curing on chloride penetration in concrete containing cement replacement materials. The results show that at early periods of exposure, initial curing has a substantial influence on chloride penetration in concrete.

* Corresponding author: 2973321850@qq.com 
The presence of temperature can cause convection of fluids, and researchers have done a lot of research on convection. Chen et al. [11] dis-cussed the mutual coupling of temperature gradients and concentration gradients in natural convection in porous media cavities and their effects on heat and mass transfer. The finite element method with penalty function was used to numerically simulate the distribution of temperature field and con-centration field of the flow field and the change of heat and mass transfer coefficients of the wall un-der four different boundary conditions. Jan et al. [12] studied the effects of unnatural natural convection of viscous fluids and heat and mass transfer at elevated wall temperatures in the case of sliding wall conditions at the boundary. Alsabery et al. [13] used a finite difference method to analyze the free convection of nanofluids in an inclined square shell with two sinusoidal temperature boundary conditions consisting of a porous layer and a nanofluid layer applied to two opposing vertical walls. The obtained results show that the heat transfer is considerably affected by the porous layer increment. Several nanoparticles depicted a diversity improvement on the convection heat transfer.

The effect of convection (laminar flow) caused by the temperature difference on chloride ion transport in concrete exists. Unfortunately, few studies have focused on the influence of laminar flow caused by the temperature difference on chloride ion transport in concrete. In view of this, this paper establishes a 2-D electrochemical chloride extraction mesoscale model, and based on Navier-Stokes equations, and the finite element method is used to simulate the non-isothermal flow coupled with chloride ion diffusion in concrete components. Based on this simulation, the effects of temperature transfer on the fluid motion and chloride ion migration in the concrete are analyzed, and the effect of convective motion of the fluid on the temperature transfer of concrete is studied.

\section{Chloride ion diffusion model}

\subsection{Basic model}

In 1970, Collepardi and others first advocated the use of Fick's second diffusion law to describe the apparent diffusion behavior of chloride ions in concrete. The basic assumptions are as follows: (1) concrete is a semi-infinite uniform medium; (2) the diffusion of concrete is onedimensional; (3) Chloride does not adsorb and combine with concrete, that is, the chloride ion binding capacity of concrete is zero; (4) The chloride ion diffusion coefficient is a constant; (5) Boundary conditions (that is, the chloride ion concentration of the exposed surface) are constant. Accordingly, Fick's second law is:

$$
\frac{\partial c}{\partial t}=D \frac{\partial^{2} c}{\partial x^{2}}
$$

where $t$ is time; $x$ is the distance from the surface; $D$ is the chloride diffusion coefficient; $c$ is the initial concentration.

\subsection{Effect of temperature on chloride diffusion coefficient}

The temperature has an effect on the migration capacity of chloride ions, and the Arrhenius equation describes the relationship between temperature and the diffusion coefficient of chloride ions [9]:

$$
D_{T}=D_{0}\left[\frac{T}{T_{1}}\right] \cdot e^{q\left[\frac{1}{T_{1}}-\frac{1}{T}\right]}
$$

where and $D_{\mathrm{T}}$ are the chloride diffusion coefficients at temperatures $T$ and; $T_{l}$ is the reference temperature; $T$ is the ambient temperature; $q$ is the activation constant and is related to the water-cement ratio. When the watercement ratio is $0.4, q$ is $6000 \mathrm{~K}$; when the water-cement ratio is $0.5, q$ is $5450 \mathrm{~K}$; when the water-cement ratio is 0.6 , $q$ is $3850 \mathrm{~K}$ [14].

\subsection{Model assumptions}

(1) The ionic diffusion coefficients in the model are the same;

(2) Concrete is a semi-infinite homogeneous medium;

(3) The diffusion of concrete is one-dimensional;

(4) Chloride does not adsorb and binds with concrete, that is, the chloride ion binding capacity of concrete is zero;

(5) The chloride diffusion coefficient is constant;

(6) Boundary conditions (i.e., chloride ion concentration on the exposed surface) are constant.

Model setup as shown in Fig. 1:

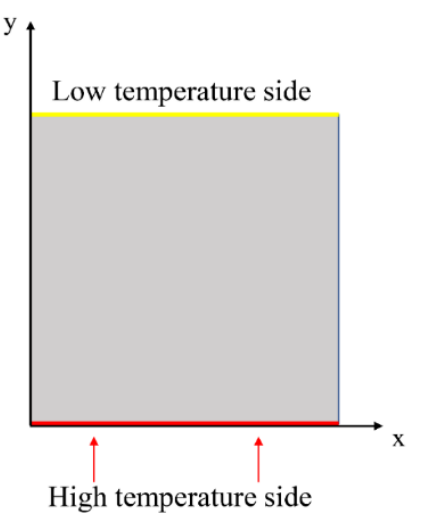

Fig. 1. Model settings.

\subsection{Model validation}

A water-cement ratio of 0.4 and a curing time of 56 days were selected for simulation. We compared the heat transfer model with experimental data [15]. The results obtained are shown in Fig. 2. As can be seen from the figure, the heat transfer model is basically in agreement with the experimental data. 


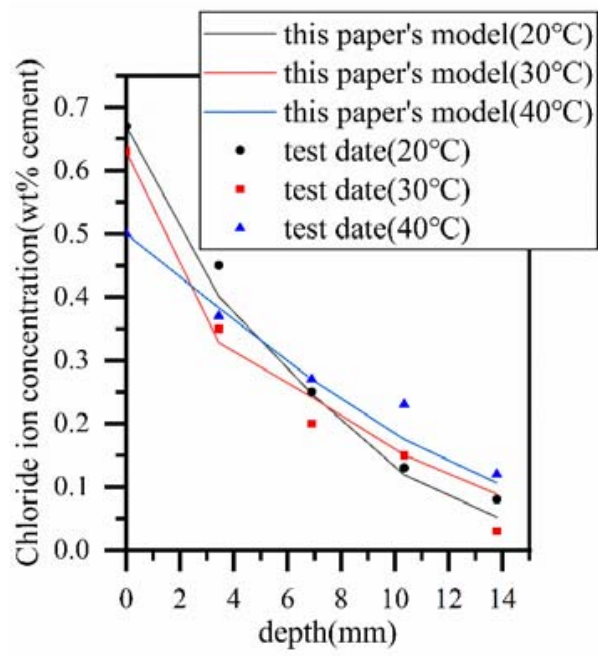

Fig. 2. Comparison of the simulation results and the test data with different temperature.

\section{Theory of laminar flow}

The Heat and mass transfer in porous media are common in nature and engineering practice and have an important impact on the actual process. In a saturated porous medium, due to the temperature inhomogeneity, the density difference of the fluid inside the medium causes buoyancy, which leads to natural convective heat and mass transfer processes [16].

\subsection{The Reynolds number}

A fundamental characteristic in analyses of fluid flow is the Reynolds number [17]:

$$
R e=\frac{\rho U L}{\mu}
$$

where $U$ denotes a velocity scale, and $\mathrm{L}$ denotes a representative length, $\rho$ is the density, $\mu$ is the dynamic viscosity. The Reynolds number represents the ratio of inertial and viscous forces. At low Reynolds numbers, viscous forces dominate and tend to damp all disturbances, resulting in laminar flow. At high Reynolds numbers, the damping of the system is very low, making small disturbances likely to increase through nonlinear interactions. If the Reynolds number is high enough, the flow field will eventually be in a chaotic state, called turbulence. It should be noted that the Reynolds number may have different meanings according to the length scale and the speed scale. To be able to com-pare two Reynolds numbers, they must be based on the equivalent length and speed ranges.

In practical engineering applications, such as oil extraction, groundwater infiltration, blood flow, or concrete materials, the Reynolds number of the in-ternal flow is small, and most can be regarded as laminar flow [18].

\subsection{General single-phase flow theory}

Fluid flow in porous media still meets continuity assumptions [18]. This means that fluid percolation in the pores of porous media can be described by Navier-Stokes equations. The Single-Phase Fluid Flow interfaces are based on the Navier-Stokes equations, which in their most general form read [19]:

$$
\begin{gathered}
\frac{\partial \rho}{\partial t}+\nabla \cdot(\rho \mathrm{u})=0 \\
\rho \frac{\partial u}{\partial t}+\rho(\mathrm{u} \cdot \nabla) \mathrm{u}=\nabla \cdot[-p \mathrm{I}+\tau]+\mathrm{F} \\
\rho C_{P}\left(\frac{\partial T}{\partial t}+(\mathrm{u} \cdot \nabla) T\right)=-(\nabla \cdot \mathrm{q})+\tau: \mathrm{S}- \\
\left.\frac{T}{\rho} \frac{\partial \rho}{\partial T}\right|_{p}\left(\frac{\partial p}{\partial t}+(\mathrm{u} \cdot \nabla) p\right)+Q
\end{gathered}
$$

where $\rho$ is the density, $u$ is the velocity vector, $p$ is pressure, $\tau$ is the viscous stress tensor, $\mathrm{F}$ is the volume force vector, $C_{\mathrm{p}}$ is the specific heat capacity at constant pressure, $Q$ contains the heat sources, $S$ is the strain-rate tensor:

$$
\mathrm{S}=\frac{1}{2}\left(\nabla \mathrm{u}+(\nabla \mathrm{u})^{T}\right)
$$

The operation ":" denotes a contraction between tensors defined by:

$$
\mathrm{a}: \mathrm{b}=\sum_{n} \sum_{m} a_{n m} b_{n m}
$$

This is sometimes referred to as the double dot product. Equation (4) is a continuous equation and repre-sents mass conservation. Equation (5) is a vector equation representing momentum conservation. Equation (6) describes the conservation of energy, formulated in terms of temperature. These equa-tions intuitively illustrate the boundary conditions. For Newtonian fluids that have a linear relationship between stress and strain, Stokes [20] derives the following formula:

$$
\tau=2 \mu \mathrm{S}-\frac{2}{3} \mu(\nabla \cdot \mathrm{u}) \mathrm{I}
$$

It can be seen from equation (9) that the dynamic viscosity $\mu$ of the Newtonian fluid is related to temperature, but not to the velocity field. In the numerical simulation in this paper, the heat transfer module considers the pore solution as Newtonian fluid, according to equation (9).

\subsection{Weakly compressible flow}

When the temperature variations in the flow are small, a single-phase fluid can often be assumed incompressible; that is, $\rho$ is constant or nearly constant. However, when there is sufficient temperature, $\rho$ is not a fixed value. At this time, we consider it as a compressible fluid or a weakly compressible fluid. The same equations as for Compressible Flow are applied for weakly compressible flow. The equations of motion for a single-phase fluid are the continuity equation [17]:

$$
\frac{\partial p}{\partial t}+\nabla \cdot(\rho \mathrm{u})=0
$$

Combine equations (7), (9) and (5) to get the momentum equation:

$$
\begin{aligned}
& \rho \frac{\partial u}{\partial t}+\rho \mathrm{u} \cdot \nabla \mathrm{u}=-\nabla p \mathrm{I}+\nabla \cdot\left(\mu\left(\nabla \mathrm{u}+(\nabla \mathrm{u})^{T}\right)-\right. \\
& \left.\frac{2}{3} \mu(\nabla \cdot \mathrm{u}) \mathrm{I}\right)+\mathrm{F}
\end{aligned}
$$

The above equation can be used as a governing equation to describe the weakly compressible flow, whose 
density and dynamic viscosity are affected by temperature.

\subsection{Non-isothermal flow and diffusion coupling solution of chloride ion}

The coupling of the chloride ion diffusion field, temperature transmission, and laminar flow module adopts the inheritance setting of the solution, that is, the chloride ion transmission changes with the change of laminar flow, and the laminar flow module also changes with the temperature When a change occurs, the solution of the previous substep is used as the initial condition of the next substep, and so on. In simulation technology, the meshing process of the established model is very important. The accuracy of the meshing directly affects the accuracy of the final simulation results. The model is a rectangular mesh near the wall, and the rest is Triangular mesh, as shown in Fig. 3.

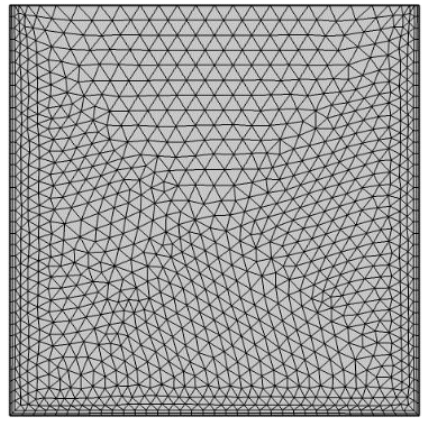

Fig. 3. Mesh division.

\section{Results and discussion}

\subsection{Effect of temperature transfer on laminar flow and chloride ion transport in saturated concrete}

We set four temperature conditions: $5{ }^{\circ} \mathrm{C}, 20^{\circ} \mathrm{C}, 40{ }^{\circ} \mathrm{C}$, and $60{ }^{\circ} \mathrm{C}$ to characterize the ambient temperature on one side (lower side) of the concrete. The laminar flow

(a)
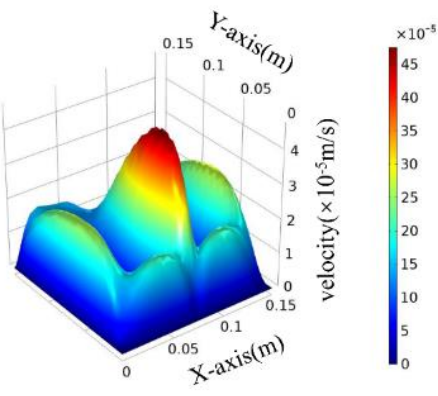

(c)

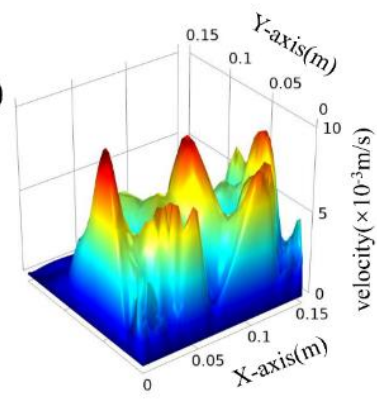

(b)

velocity diagram for simulating heat transfer for 12 hours under these four temperature conditions is shown in Fig. 4. It can be seen from the figure that the laminar flow velocity inside the concrete increases with increasing temperature. The reason is that the increase in temperature causes the dynamic viscosity of the pore solution to decrease. Dynamic viscosity $\mu$ describes the relationship between the shear rate and the shear stress in the fluid. Intuitively, water and air have low viscosities and are often described as thicker substances (such as oil) with higher viscosities. The dynamic viscosity of water is related to temperature. The internal energy of water increases with increasing temperature, and the viscosity coefficient of water decreases with increasing temperature, which is consistent with the findings of other researchers.[21].

Taking C50 concrete as an example, $\mathrm{w} / \mathrm{b}=0.35$, linear regression is performed according to the recommended value of formula (2), and its $\mathrm{q}$ value is $6712 \mathrm{~K}$. The chloride ion concentration distribution in the concrete within 12 hours of corrosion was obtained by simulation. The results are shown in Fig. 5. It can be seen from the figure that as the temperature rises, the penetration rate of chloride ions in the concrete increases significantly; when the temperature reaches $60{ }^{\circ} \mathrm{C}$, the chloride ion transmission speed is the fastest; when the concrete is in a low- temperature environment, its chloride ion transmission speed decreases significantly. In order to further explore the effect of different temperature transfers on the chloride ion transport in the concrete, the evolution of the chloride ion concentration in the middle of the concrete (abscissa: $75 \mathrm{~mm}$ ) was selected for analysis. The results are shown in Fig. 6. It can be observed from the figure that the chloride ion concentration of concrete gradually decreases with increasing depth, and gradually stabilizes at a certain depth. Numerical simulations will reflect the chloride diffusion behavior in concrete. As the temperature increases, the chloride ion concentration at the same depth of the concrete increases, and its longitudinal transmission distance increases significantly.

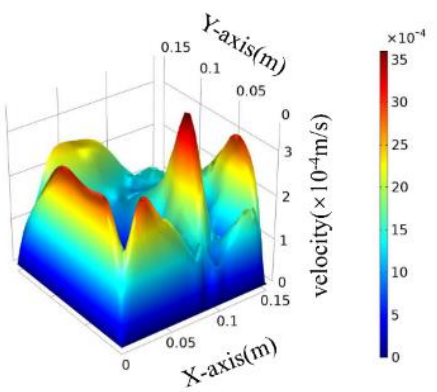

(d)

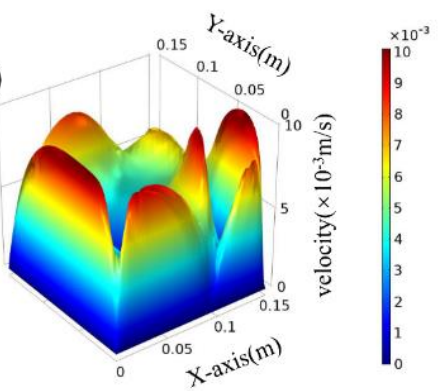

Fig. 4. Effect of temperature on laminar flow rate within 12 hours (a) $5{ }^{\circ} \mathrm{C}$, (b) $20{ }^{\circ} \mathrm{C}$, (c) $40{ }^{\circ} \mathrm{C}$, (d) $60{ }^{\circ} \mathrm{C}$. 
(a)

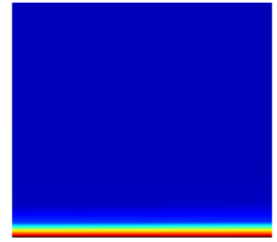

(c)

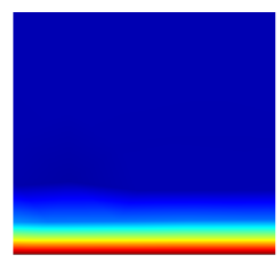

(b)

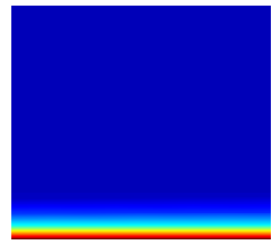

(d)

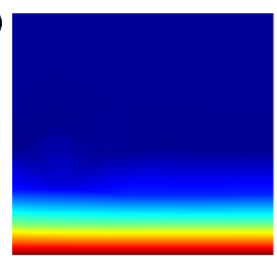

Fig. 5. Chloride ion transmission diagrams in concrete under different temperature conditions without considering laminar flow in 12 hours (a) $5{ }^{\circ} \mathrm{C}$, (b) $20^{\circ} \mathrm{C}$, (c) $40{ }^{\circ} \mathrm{C}$, (d) $60{ }^{\circ} \mathrm{C}$.

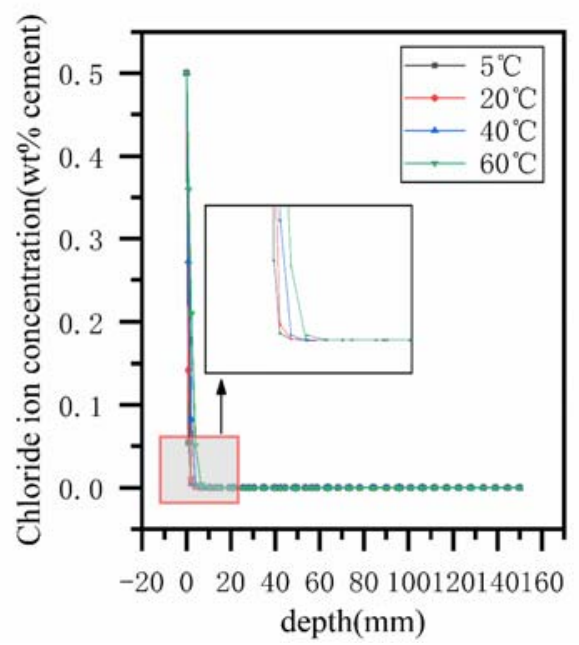

Fig. 6. Effect of non-isothermal flow heat transfer on chloride ion concentration in concrete.

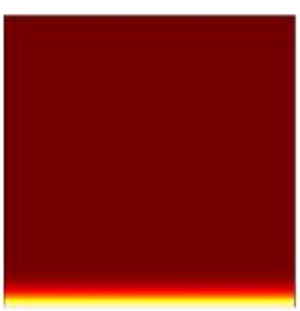

No laminar flow

(a)

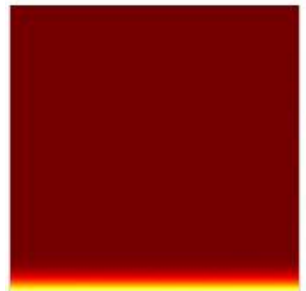

No laminar flow

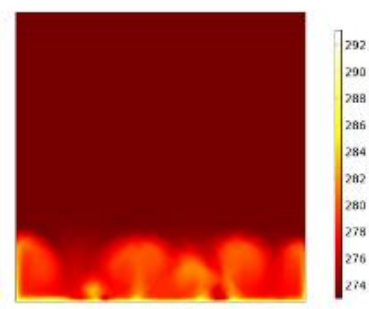

Laminar flow

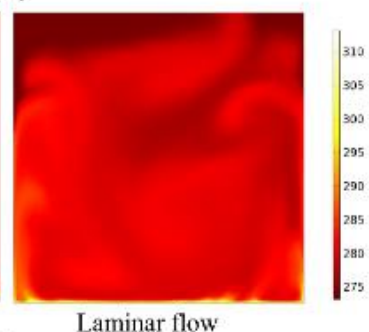

(c)
Laminar flow

\subsection{Effect of laminar flow on temperature transmission of saturated concrete}

In order to accurately describe the temperature transmission in the concrete, the first 3 minutes of the heat source work was selected. After the pore solution was heated at the bottom of the concrete, the local density decreased, which caused the internal flow of the concrete, and the temperature was affected by it, as shown in Fig. 7. It can be clearly seen from the cloud diagram that when the effect of laminar flow is considered, the temperature is transmitted faster in the concrete, and the phenomenon of uneven diffusion from high temperature to low temperature appears, which is consistent with the second law of thermodynamics. The reason for the uneven heat transfer may be that the macroscopic movement of the fluid causes relative displacement between the various parts of the fluid, and the mixing of cold and hot fluids causes convective heat transfer. In addition, it can be observed that the effect of laminar flow on the heat transfer of concrete increases with increasing temperature, which is mutually corroborated by the description of laminar flow velocity in Section 4.1, that is, the higher the temperature, the faster the laminar velocity and the more intense the convection.

In order to more intuitively observe the effect of laminar flow on the heat transfer of concrete, a $3 \mathrm{~min}$ isotherm diagram is obtained, as shown in Fig. 8. In general, the isotherms are dense, and the temperature difference is large; the isotherms are sparse, and the temperature difference is small. It can be seen from the figure that when the temperature is high, the isotherms on both sides of the concrete are dense, indicating that the temperature changes here are large, presumably because the higher temperature causes greater uneven heat transfer, and the internal space of the concrete test block is relatively large. Small, both sides are limited by "walls," so more intense temperature transmission moves along the walls to lower temperatures.

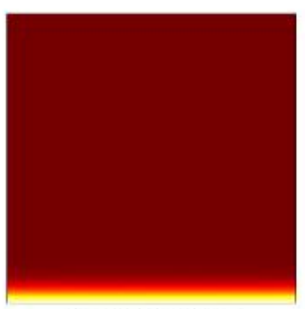

No laminar flow

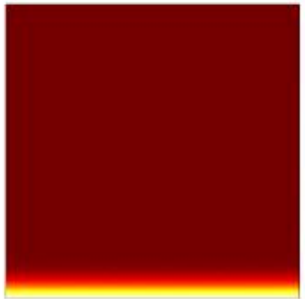

No laminar flow

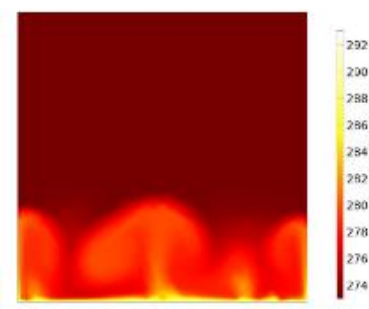

Laminar flow

(b)

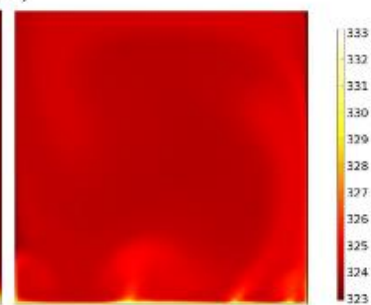

(d)

Laminar flow ,

Fig. 7. Effects of laminar flow on the internal temperature diffusion of concrete at different ambient temperatures (a) $5^{\circ} \mathrm{C}$, (b) $20^{\circ} \mathrm{C}$, (c) $40^{\circ} \mathrm{C}$, (d) $60^{\circ} \mathrm{C}$. 
(a)

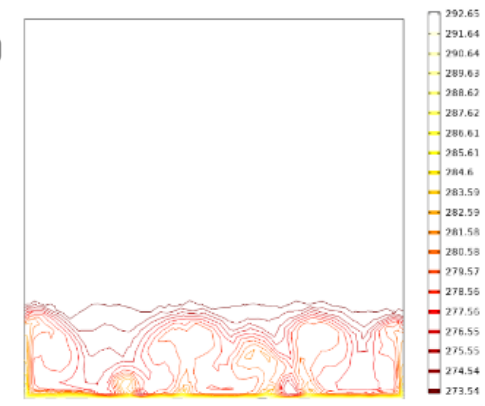

(c)

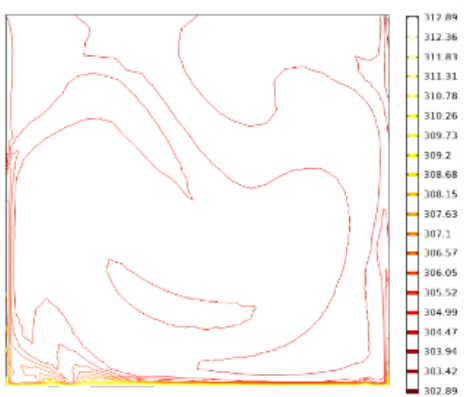

(b)

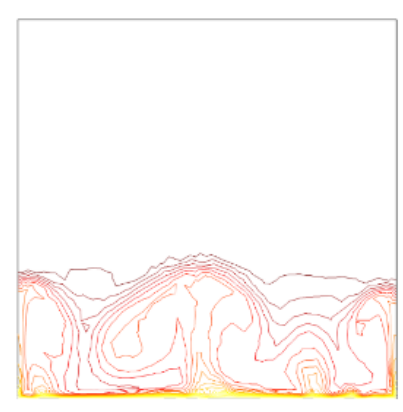

(d)

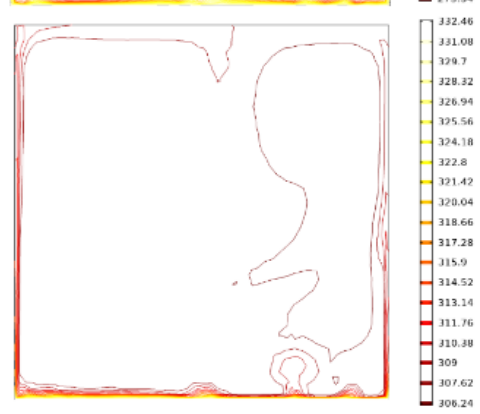

Fig. 8. Temperature contours at different ambient temperatures (a) $5{ }^{\circ} \mathrm{C}$, (b) $20{ }^{\circ} \mathrm{C}$, (c) $40{ }^{\circ} \mathrm{C}$, (d) $60{ }^{\circ} \mathrm{C}$.

\subsection{Effect of non-isothermal heat transfer (heat transfer considering laminar flow) on chloride ion transport in saturated concrete}

Laminar flow is generated at non-uniform temperature. When considering laminar flow, the effect of temperature on chloride ion transport is shown in Fig. 9. By comparing Figures 5 and 9, we find it difficult to visually observe the effect of non-isothermal flow on chloride ion transport. This simulation considers saving the calculation time and simplifying the calculation, and only calculates the 12hour chloride ion transmission process. It is well known that the transmission of chloride ions in concrete is a slow process, and only a small part is transmitted in 12 hours. And laminar flow can drive rapid temperature transmission, which in essence is fast energy transmission, and the diffusion of chloride ions will not be affected so much by laminar flow.

However, the 12-hour period is sufficient to reflect the tendency of non-isothermal flow to affect chloride ions. In order to further explore the effect of non-isothermal flow on the chloride ion transport inside the concrete, the evolution of the chloride ion concentration in the middle of the concrete (abscissa 75) was selected for analysis, and the results are shown in Figure 10. It can be seen from Fig. 10 that non-isothermal flow will promote the diffusion of chloride ions in the concrete, and the higher the temperature, the more obvious the effect of laminar flow on it. The reason is that the increase in temperature reduces the dynamic viscosity of the fluid, which causes the non-isothermal flow speed to increase, which drives the chloride ions to transport inward. It is worth noting that despite the impact, it is not obvious. The reason is that the 12-hour time is very short compared to the chloride ion penetration process in the concrete, but it is sufficient to describe the trend of the impact. In the environment, over time, the effect of non-isothermal flow on chloride ion transport in concrete cannot be ignored. (a)

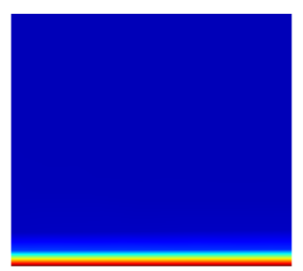

(c)

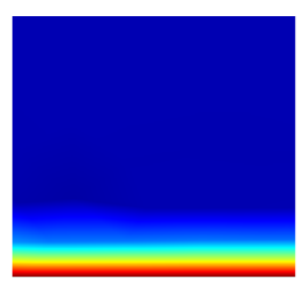

(b)

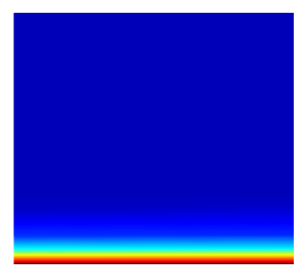

(d)

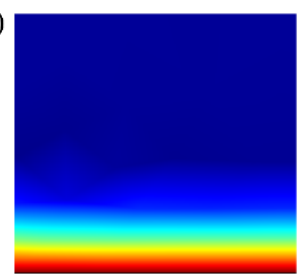

Fig. 9. Cloud diagrams of chloride ion transmission in concrete under different temperature conditions considering laminar flow (a) $5{ }^{\circ} \mathrm{C}$, (b) $20{ }^{\circ} \mathrm{C}$, (c) $40{ }^{\circ} \mathrm{C}$, (d) $60{ }^{\circ} \mathrm{C}$. 

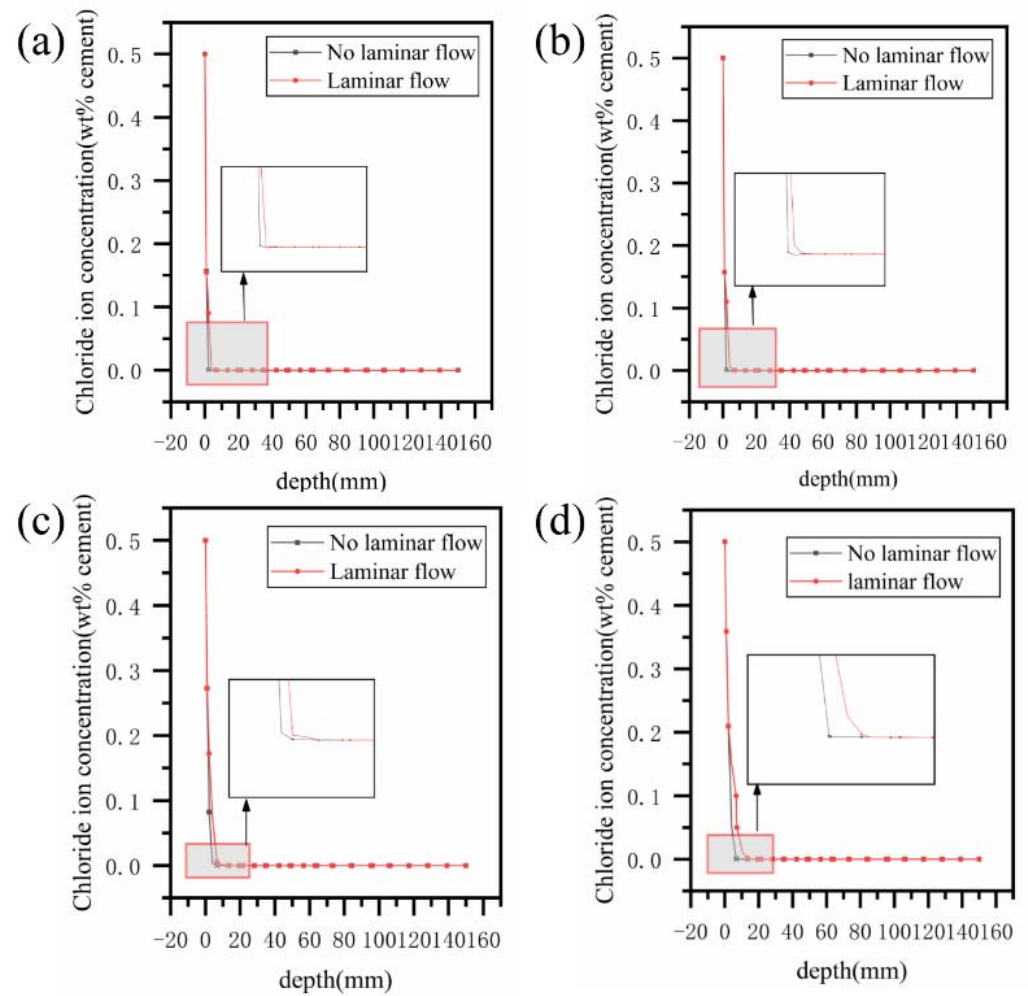

Fig. 10. Effects of laminar flow on chloride ion transport in concrete under different temperature conditions (a) $5{ }^{\circ} \mathrm{C}$, (b) $20{ }^{\circ} \mathrm{C}$, (c) $40{ }^{\circ} \mathrm{C}$, (d) $60{ }^{\circ} \mathrm{C}$.

\section{Conclusion}

In this paper, a meso-scale model of chloride ion transport affected by temperature and laminar flow in nonisothermal flow is developed and compared with the measured data. At the same time, based on the finite element software COMSOL, the effect of non-isothermal flow on the diffusion of chloride ions inside the concrete was analyzed, and the effect of laminar flow on temperature transmission was studied. The following conclusions are made based on the numerical simulation results:

(1) When the heating time is the same, the dynamic viscosity of the fluid in the concrete decreases with increasing temperature; in other words, the temperature and the laminar flow velocity are positively correlated.

(2) As the temperature increases, the chloride ion concentration at the same depth of the concrete increases, and its longitudinal transmission distance increases significantly. If the influence of temperature on the chloride ion transmission of concrete is not considered, it will affect the accuracy of concrete life assessment.

(3) When the effect of laminar flow is considered, the temperature is transmitted faster in the concrete, and the phenomenon of uneven diffusion from high temperature to low temperature appears. The effect of laminar flow on the heat transfer of the concrete increases with increasing temperature

(4) The non-isothermal flow will promote the diffusion of chloride ions in concrete, and the higher the temperature, the more obvious the effect of laminar flow on it.

This article only considers the non-isothermal flow in saturated concrete. When the concrete is in an unsaturated state, due to the lack of internal moisture, the chloride ion transmission rate decreases, at this time, the effect of convection on the chloride ion transmission in the concrete will be affected in future work the study.

\section{Acknowledgments}

The authors wish to acknowledge the financial support from Postgraduate Research \& Practice Innovation Program of Jiangsu Province (No. SJCX20_1477), Jiangsu Higher Education Institutions of China (No. 19KJB560004)

\section{References}

1. J. Xu, F.M. Li, J. Zhao, L. Huang, Model of timedependent and stress-dependent chloride penetration of concrete under sustained axial pressure in the marine environment, Construction and Building Materials. 170:207-216. (2018)

2. R.R. Yin, C. Zhang, Q. Wu, B. Li, H. Xie, Damage on lining concrete in highway tunnels under combined sulfate and chloride attack, Frontiers of Structural and Civil Engineering.12(3): 331-340 (2018)

3. C. Yu, Q. Wu, J.M. Yang, Effect of seawater for mixing on properties of potassium magnesium phosphate cement paste, Construction and Building Materials.155:217-227 (2017)

4. H. Fan, P.G. Wang, T.J. Zhao, Study on Chloride Ion Intrusion into Concrete Structure after Long-term 
Expo-sure. Journal of Building Structures 32(1): 8894 (2011)

5. L. Hou, Z. Ye, B. Zhou, C. Shen, D. Chen, Bond behavior of reinforcement embedded in steel fiber reinforced concrete under chloride attack. Struct Concrete (2019)

6. Y. Abbas, F. Pargar, D.A. Koleva, van. K. Breugel, W. Olthuis, A. van. Den. Berg, Non-destructive measurement of chloride ions concentration in concrete-A comparative analysis of limitations and prospects, Construction and Building Materials. 174:376-387 (2018)

7. T.V. Quan, A. Soive, Baroghel-Bouny, V.. Modelisation of chloride reactive transport in concrete including thermodynamic equilibrium, kinetic control and surface complexation. Construction and Building Materials 110: 70-85 (2018)

8. L.C. Wang, T. Ueda, T, Meso-scale modeling of chloride diffusion in concrete with consideration of effects of time and temperature. Water Science and Engineering 2(3): 58-70 (2009)

9. R. Zhao, Z.Q. Jin, J.R Cao, F.Y. Du, Y.Kang, Study on the Change of Temperature and Humidity in the Marine Environment to Chloride Transmission of Concrete. The Ocean Engineering (in Chinese) 36(1): 99-106 (2018)

10. J.M. Khatib, P.S. Mangat, Influence of hightemperature and low-humidity curing on chloride pene-tration in blended cement concrete. Cement and Con-crete Research 32(11): 1743-53 (2002)

11. B.M. Chen, B.X. Wang, Z.H. Fang, Coupling of temperature gradient and concentration gradient in natural convection in porous media. Journal of Engineering Thermophysics (02): 210-214 (1995)

12. S.U. Jan, S.U. Haq, S.I.A. Shah, I. Khan, M.A. Khan, General Solution for Unsteady Natural Convection Flow with Heat and Mass in the Presence of Wall Slip and Ramped Wall Temperature. Communications in Theoret-ical Physics 71(6): 647 (2019)

13. A.I. Alsabery, A.J. Chamkha, H. Saleh, I. Hashim, Natural Convection Flow of a Nanofluid in an Inclined Square Enclosure Partially Filled with a Porous Medium. Nature 7(1): 2357 (2017)

14. L.Y. Li, J. Xia, S.S. Lin, A multi-phase model for predicting the effective diffusion coefficient of chlorides in concrete, Construction and Building Materials. 26(1):295-301 (2012)

15. H.C. Yang, A.M. Du, Z.H. Fan, J.B. Xiong, S.N. Wang, Effect of temperature on chloride ion diffusion proper-ties of concrete. Prot \& Waterway Engineering. (10): 20-6 (2015)

16. F. Liu, B.M. Chen, L. Wang, Effect of Porous Media on Convective Heat and Mass Transfer in a Closed Cavi-ty. Journal of Shandong University (Engineering Sci-ence) 41(1): 145-50 (2011)

17. G.K. Batchelor, An Introduction to Fluid Dynamics: Cambridge University Press (1967)

18. Y.P. Huang, C.B. Zhang, Numerical simulation of seepage behavior in porous media. Building Energy En-vironment 35(4): 38-42 (2016)

19. P. M. Gresho, R.L. Sani, Incompressible Flow and the Finite Element Method, Volume 2: Isothermal Laminar Flow: John Wiley \& Sons (2000)

20. G.G. Stokes, Trans: Camb. Phil. Soc 8: 287-305 (1845)

21. Y.M. Lou, J.H. Liu, X.P. Zhou, J.C. Liu, Study on the Influence of Temperature on Water Viscosity and Diffu-sion Coefficient. Journal of Southwest China Normal University (Natural Science Edition) 34(6): 34-9 (2009) 